\title{
Sequent of Relations Calculi: A Framework for Analytic Deduction in Many-Valued Logics
}

\author{
Matthias Baaz, A. Ciabattoni`, Christian G. Fermüller \\ Technische Universität Wien \\ A-1040 Vienna, Austria \\ [baaz, agata, chrisf] @logic .at
}

\begin{abstract}
We present a general framework that allows to construct systematically analytic calculi for a large family of (propositional) many-valued logics - called projective logics — characterized by a special format of their semantics. All finite-valued logics as well as infinite-valued Gödel logic are projective. As a case-study, sequent of relations calculi for Gödel logics are derived. A comparison with some other analytic calculi is provided.
\end{abstract}

\section{Introduction}

A central task of logic in computer science is to provide analytic - "Gentzenstyle" - calculi for a wide range of non-classical logics. Such calculi serve as a basis for automated deduction and allow the extraction of more information from proofs (compared to traditional Hilbert-style systems). A large number of Gentzen-style systems for many-valued logics have been introduced since the 1950s (see, e.g., the handbook article [9] for an overview). In particular, it is now well understood how to construct efficient analytic proof systems in a uniform manner for the whole family of finite-valued logics, using manyplaced (or labeled) sequents or, equivalently, signed tableaux. These systems not only allow to handle connectives defined by arbitrary finite matrices, but have also been extended to so-called distribution quantifiers, a rather general concept of quantification in many-valued logic. However, in contrast to the finite-valued case, there is much less literature on analytic Gentzenstyle systems for infinite-valued logics. In particular, many important infinitevalued logics, such as two of the three fundamental formal representations of fuzzy logic — namely Łukasiewicz [21] and Product logic [20] — still seem to escape satisfactory characterizations through elegant and useful analytic proof systems. An important exception is the third main formalization of fuzzy logic, namely Gödel logic $\mathrm{G}_{\infty}$. Analytic systems for this logic — also known as (Gödel-)Dummett logic, since Dummett [14] presented the first axiomatization matching Gödel's matrix characterization - can be found, e.g., in $[25,1-3,16,4,5]$. The interest in $\mathrm{G}_{\infty}$ is well motivated by the fact that

\footnotetext{
* Research supported by EC Marie Curie fellowship HPMF-CT-1999-00301.
} 
it naturally turns up in a number of different contexts. Already in the 1930s Gödel [18] used it in investigations of intuitionistic logic; later, Dunn and Meyer [15] pointed out its relevance for relevance logic; Visser [29] employed it in investigations of the provability logic of Heyting arithmetic; and eventually it was recognized as one of the most important formal representations of fuzzy logic. In particular, the connectives of $\mathrm{G}_{\infty}$ correspond to the min-max $t$-norm and its residuum (see [19]).

The purpose of this contribution is not just to introduce yet another analytic proof system for $\mathrm{G}_{\infty}$, but to present a general framework that allows to construct analytic calculi for a large family of (propositional) many-valued logics, including $G_{\infty}$ as a prime example. Our calculi - called sequents of relations - are systematically derived from a specific presentation of the semantics of the connectives involved. This format of presentation inspires us to call the corresponding class of logics "projective". Our main example of a projective logic is $\mathrm{G}_{\infty}$. But also $\mathrm{G}_{\infty}^{\Delta}$, i.e., $\mathrm{G}_{\infty}$ enriched with the $\Delta$ projection modality introduced in [22] (see also [6]), as well as all finite-valued logics can be considered projective, as we will explain. In particular, this allows us to transfer the obvious semantic connection between $\mathrm{G}_{\infty}$ and the finite-valued Gödel logics to corresponding proof systems.

The main concepts and results of this paper summarize the two conference contributions [8] and [7].

The paper is organized as follows: in Section 2 we define the class of projective logics and we show that the family of (finite- and infinite-valued) Gödel logics is a particular case of them (see Section 2.1). Section 2.2 relates finite- and infinite-valued projective logics. Sequents of relations calculi are introduced in Section 3. There we also shown how to translate a given specification of a projective logic into such a calculus in a systematic, even mechanizable, way. Soundness and completeness of the obtained calculi is proved in Section 3.1. Admissibility of cut rules as well as of other structural rules in sequent of relations calculi is discussed in Section 3.2. The special case of Gödel logics is presented in detail in Section 4. Sequent of relations calculi for finite and infinite-valued Gödel logic, as well as $G_{\infty}^{\Delta}$, are derived. Relationships between sequents of relations and some other analytic calculi for Gödel logics are discussed in Section 4.1.

\section{Projective logics}

The syntax of the propositional logics considered here is very general. The (object) language for a logic is based on an infinite supply of propositional variables, a finite set of connectives (with fixed arity), and a finite number of truth constants. (Truth constants will also be considered as 0-ary connectives.) As usual, the formulas of such a language are build up from its variables, constants and connectives. 
The logics under investigation are characterized by a special format of the definition of their semantics. Again, we take a very general approach. To specify a semantics we refer to some (classical, first order) theory $\mathbf{T}$ called semantic theory - whose intended range of discourse is a set of truth values $V$. T can, e.g., be the theory of linear orders or lattices or any other class of relational structures. It can also be the (first order) specification of a single structure. The only requirements we put on $\mathbf{T}$ are as follows:

(F) $\mathbf{T}$ is based on a function free language with finite signature. I.e., the atomic formulas are of form $R\left(t_{1}, \ldots, t_{k}\right)$, where the $t_{i}$ are variables or constants.

(D) The set of $\Pi_{1}$-formulas that are valid in $\mathbf{T}$ is decidable.

We use the notation " $\mathcal{M} \models A[\sigma]$ " to denote that the formula $A$ is satisfied in a model $\mathcal{M}$ (of $\mathbf{T}$ ) under the assignment $\sigma$ of elements of the domain of $\mathcal{M}$ to the free variables of $A$. The domain of $\mathcal{M}$ is called set of truth values. By " $\mathbf{T} \models A$ " we mean that $A$ is valid in $\mathbf{T}$, i.e. $A[\sigma]$ is satisfied in all models of $\mathbf{T}$ for all assignments $\sigma$.

Constants of $\mathbf{T}$ denote truth values and are identified with truth constants of the object language.

Quantifier and negation free formulas of $\mathbf{T}$, i.e., formulas built up from atomic formulas using conjunction and disjunction only, will

play a special rôle. Let us call such formulas simple.

We call an $n$-ary connective $\square$ projective (with respect to an interpretation based on a semantic theory $\mathbf{T}$ ) if the corresponding truth function $\widetilde{\square}$ can be written in the following form:

$$
\widetilde{\square}\left(x_{1}, \ldots, x_{n}\right)= \begin{cases}t_{1} & \text { if } A_{1} \\ \vdots & \vdots \\ t_{m} & \text { if } A_{m}\end{cases}
$$

where each $t_{i}$ is either a truth constant or in $\left\{x_{1}, \ldots, x_{n}\right\}$. Moreover, the conditions $A_{i}$ are simple formulas of the underlying semantic theory $\mathbf{T}$ whose free variables are among $\left\{x_{1}, \ldots, x_{n}\right\}$. Since $\tilde{\square}$ is a total function these conditions have to satisfy the following properties:

Totality: $\mathbf{T} \models \forall x_{1} \cdots \forall x_{n} \bigvee_{1 \leq i \leq m} A_{i}$

Functionality: for all $i \neq j ; i, j \in\{1, \ldots, m\}: \mathbf{T} \mid=\forall x_{1} \cdots \forall x_{n} \neg\left(A_{i} \wedge A_{j}\right)$

To specify a logic we also need a notion of designated truth values or, shorter, designating predicate. Any simple formula Des $(x)$ of $\mathbf{T}$ with exactly one free variable $x$ may be chosen for this purpose. ${ }^{1}$

\footnotetext{
${ }^{1}$ If one prefers to exclude the empty logic and the inconsistent "top logics" consisting of all formulas of a particular signature - from the realm of logics then one should insist on $\mathbf{T} \models \exists x \operatorname{Des}(x)$ and $\mathbf{T} \models \exists x \neg \operatorname{Des}(x)$, respectively.
} 
In general, for a propositional many-valued logic, an interpretation $\mathcal{I}$ is a mapping from the set of propositional variables $\mathcal{P} \mathcal{V}$ into some set $V$ of truth values. Given truth functions $\widetilde{\square}$ for all connectives $\square$ of the language, an interpretation $\mathcal{I}$ extends to an evaluation function val $_{\mathcal{I}}$, that maps all formulas into truth values, as follows:

$$
\begin{gathered}
\operatorname{val}_{\mathcal{I}}(F)=\mathcal{I}(F) \quad \text { if } F \in \mathcal{P} \mathcal{V} \\
\operatorname{val}_{\mathcal{I}}\left(\square\left(F_{1}, \ldots, F_{n}\right)\right)=\widetilde{\square}\left(\operatorname{val}_{\mathcal{I}}\left(F_{1}\right), \ldots, \operatorname{val}_{\mathcal{I}}\left(F_{n}\right)\right)
\end{gathered}
$$

Note that this definition is independent of the particular semantic framework described above.

In the context of a semantic theory $\mathbf{T}$, logics arise at two different levels:

(1) The set of truth values is understood as the domain of a model of $\mathbf{T}$. The semantics depends on the corresponding interpretation of the conditions $A_{i}$ of the (projective) truth functions. In this sense, any model $\mathcal{M}$ of $\mathbf{T}$ determines a logic $\mathcal{L}_{\mathcal{M}}$ : the projective logic of $\mathcal{M}$ (with respect to given projective truth functions for all connectives of the language). We call a formula $F$ valid in $\mathcal{L}_{\mathcal{M}}$ if for all interpretations $\mathcal{I}$ the designating predicate is satisfied in $\mathcal{M}$ whenever the value of $F$ is assigned to the only free variable of Des. $\mathcal{L}_{\mathcal{M}}$ is identified with the set of valid formulas, i.e.:

$$
\mathcal{L}_{\mathcal{M}}=\left\{F \mid \mathcal{M} \models \operatorname{Des}\left[\operatorname{val}_{\mathcal{I}}(F) / x\right] \text { for all } \mathcal{I}\right\}
$$

(2) There is yet another useful interpretation of this semantic machinery under which the semantic theory itself determines a logic. Namely, instead of evaluating the conditions $A_{i}$ in a particular model of $\mathbf{T}$, we may check whether the relevant instances $A_{i}^{\prime}$ of $A_{i}$ and $D e s^{\prime}$ of Des are satisfied in all models of $\mathbf{T}$. This way we do not have to fix the set of truth values in speaking of the projective logic $\mathcal{L}_{\mathbf{T}}$ associated with $\mathbf{T}$ and some projective connectives (possibly including truth constants). This allows us to speak, e.g., of the projective logic of, say, partial orders (with respect to a fixed set of projective connectives). Formally,

$$
\mathcal{L}_{\mathbf{T}}=\left\{F \mid \mathcal{M} \models \operatorname{Des}\left[\operatorname{val}_{\mathcal{I}}(F) / x\right] \text { for all } \mathcal{I} \text { and } \mathcal{M} \text { of } \mathbf{T}\right\}
$$

Example 1. To see that every finite-valued propositional logic is projective we only have to consider monadic semantic theories.

Let the language of $\mathbf{T}$ contain a monadic predicate symbol $C_{i}$ for each truth value $c_{i}$. In addition, we take the truth values as constants of the language. For any $n$-valued logic, $\mathbf{T}$ can be axiomatized by:

$$
\begin{aligned}
& \forall x: \bigvee_{1 \leq i \leq n} C_{i}(x) \\
& \text { for all } i \neq j, 1 \leq i, j \leq n: \forall x: \neg\left(C_{i}(x) \wedge C_{j}(x)\right) \\
& \text { for all } i, 1 \leq i \leq n: C_{i}\left(c_{i}\right)
\end{aligned}
$$


Any particular $n$-valued logic is determined by truth tables for all connectives and a choice of designated truth values, as usual. Every entry

$$
\widetilde{\square}\left(c_{i_{1}}, \ldots, c_{i_{n}}\right)=c_{j}
$$

in the truth table for the $n$-ary connective $\square$ translates into the part

$$
\widetilde{\square}\left(x_{1}, \ldots, x_{n}\right)=c_{j} \quad \text { if } C_{i_{1}}\left(x_{1}\right) \wedge \ldots \wedge C_{i_{n}}\left(x_{n}\right)
$$

of the definition of the truth function as above. If $\left\{c_{d_{1}}, \ldots, c_{d_{m}}\right\}$ is the set of designated truth values, obviously $C_{d_{1}}(x) \vee \ldots \vee C_{d_{m}}(x)$ serves as designating predicate. It is easy do see that here $\mathcal{L}_{\mathbf{T}}=\mathcal{L}_{\mathcal{M}}$, for every model $\mathcal{M}$ of $\mathbf{T}$.

Alternatively, we can base the semantics on a theory containing only the equality predicate and all truth constants: just replace $C_{i}(t)$ by $c_{i}=t$. This approach obviously results in the same logics as above, but excludes interpretations based on "non-intended" sets of truth values with cardinality $>n$ for $n$-valued logics.

As we shall see below, even in the case of finite-valued logics, it may be advantageous to choose a more expressive semantic theory to define the truth functions for its connectives.

\subsection{Gödel logics}

Our main example of projective logics is the family of Gödel logics. To formulate their semantics, we assume the set of truth values to be linearly ordered and equipped with a minimal element 0 and a maximal element 1 (distinct from 0). A standard axiomatization of the corresponding semantic theory is given by:

$$
\begin{array}{llll}
\forall x: \neg(x<x) & \left(\text { Irrefl }_{<}\right) & \forall x: x=0 \vee 0<x & \left(\operatorname{Min}_{<}\right) \\
\forall x \forall y \forall z:(x<y \wedge y<z) \supset x<z & \left(\text { Trans }_{<}\right) & \forall x: x=1 \vee x<1 & \left(\operatorname{Max}_{<}\right) \\
\forall x \forall y: x=y \vee x<y \vee y<x & \left(\text { Linear }_{<}\right) & 0<1 & \text { (Distinct) }
\end{array}
$$

Although one could derive a "sequent of relations calculus" (see Section 3) directly from this theory we prefer an alternative formulation of it. We do not want to have to consider "=" as a basic relation, but rather base the semantics theory, denoted by $\mathbf{T}_{\leq,<}$, on the relation symbols " $\leq$" and " $<$" by adding the following to the axioms Irrefl $<_{<}$, Trans $<$, and Distinct:

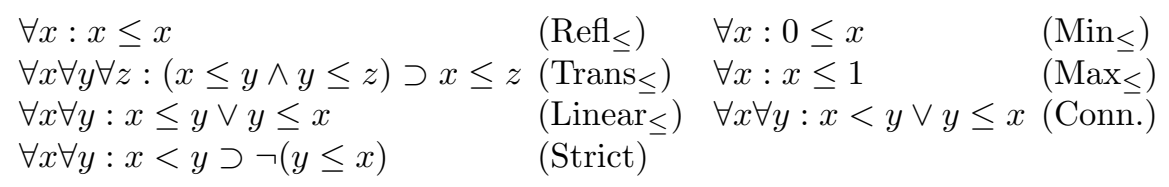

Remark 1. Efficient decision procedures for $\mathbf{T}_{\leq,<}$can be found, e.g., in [11, 12]. These results, in particular, imply that $\mathbf{T}_{\leq,<}$fulfills condition (D) on semantic theories (i.e., decidability for $\Pi_{1}$-formulas). 
We can now state the truth functions for disjunction $(\vee-$ maximum), conjunction $(\wedge-$ minimum), implication $(\supset)$, and negation $(\neg)$ in such a way that it gets clear that these connectives are projective with respect to $\mathbf{T}_{\leq,<}$.

$$
\begin{array}{ll}
\tilde{\vee}(x, y)= \begin{cases}x & \text { if } y \leq x, \\
y & \text { if } x<y\end{cases} & \tilde{\wedge}(x, y)= \begin{cases}x & \text { if } x \leq y, \\
y & \text { if } y<x\end{cases} \\
\widetilde{\supset}(x, y)= \begin{cases}1 & \text { if } x \leq y, \\
y & \text { if } y<x\end{cases} & \widetilde{\sim}= \begin{cases}1 & \text { if } x \leq 0, \\
0 & \text { if } 0<x\end{cases}
\end{array}
$$

Negation can be treated as derived connective by defining $\neg A:=A \supset 0$.

" 1 " is intended to be the only designated truth value. Therefore we take " $1 \leq x$ " as designating predicate.

Infinite-valued Gödel logic $\mathrm{G}_{\infty}$ is the logic of models of $\mathbf{T}_{\leq,<}$, that contain infinite "<-chains". This condition on models can be enforced by adding to $\mathbf{T}_{\leq,<}$the density axiom:

$$
\forall x \forall y \exists z: x<y \supset(x<z \wedge z<y) \quad \text { (Dense) }
$$

However, $\mathrm{G}_{\infty}$ is not only the logic ${ }^{2}$ of the theory axiomatized by $\mathbf{T}_{\leq,<}$extended with (Dense), or the logic $\mathcal{L}_{\mathcal{M}}$ for any dense infinite model $\mathcal{M}$ of $\mathbf{T}_{\leq,<}$, but also the logic $\mathcal{L}_{\mathbf{T}_{\leq,<}}$itself.

If we restrict attention to finite models of $\mathbf{T}_{\leq,<}$we obtain

the family of finite-valued Gödel logics $\mathrm{G}_{n}$. Let, e.g., $\mathcal{M}$ be the (up to isomorphism) unique model of $\mathbf{T}$ with 4 elements, in which also $\exists x \exists y: 0<$ $x \wedge x<y \wedge y<1$ is satisfied, then $\mathcal{L}_{\mathcal{M}}$ is the 4 -valued Gödel logic $\mathrm{G}_{4}$. Instead of focusing directly on particular models $\mathcal{M}$ one may equivalently augment $\mathbf{T}_{\leq,<}$to become the unique (first order) theory of $\mathcal{M}$. An even simpler way to obtain finite-valued Gödel logics consists in adding the following axiom to $\mathbf{T}_{\leq,<\text {: }}$

$$
\exists x_{1} \cdots \exists x_{n} \forall y: y \equiv x_{1} \vee \ldots \vee y \equiv x_{n} \quad\left(\text { Finite }_{n}\right)
$$

where $x \equiv y$ abbreviates $(x \leq y \wedge y \leq x)$. This results in $\mathcal{L}_{\mathbf{T}_{\leq,<}}=\mathrm{G}_{n}$.

Remark 2. One might intend to include conditions $\neg\left(x_{i} \equiv x_{j}\right)$, for $i \neq j$, in Finite ${ }_{n}$; however, since $G_{m}$ contains $G_{n}$ if $m<n$, these conditions are redundant.

\subsection{A relation between finite- and infinite-valued logics}

It is well known that $G_{\infty}$ is the intersection of all finite-valued Gödel logics. The concept of projective connectives allows us to grasp the connection between logics corresponding to finite and arbitrary models of a semantic theory, respectively, at a more general level.

\footnotetext{
$\overline{2}$ There is only one infinite-valued propositional Gödel logic. On the first order level different topologies on the set of truth values induce different logics.
} 
Proposition 1. Let $\mathbf{T}$ be a universal theory (i.e., axiomatized by $\Pi_{1}$-formulas only) and $F$ be any formula of a projective logic over $\mathbf{T}$. If a formula $F$ is valid in $\mathcal{L}_{\mathcal{M}}$ for all finite models $\mathcal{M}$ of $\mathbf{T}$ then $F$ is valid in $\mathcal{L}_{\mathcal{M}}$ for all models $\mathcal{M}$ of $\mathbf{T}$. More concisely:

$$
\left(\bigcap_{\mathcal{M} \text { finite }} \mathcal{L}_{\mathcal{M}}\right)=\mathcal{L}_{\mathbf{T}}
$$

Proof. Let $\mathcal{M}$ be an arbitrary model of $\mathbf{T}$ such that $\mathcal{M} \not \models \operatorname{Des}\left[\operatorname{val}_{\mathcal{I}}(F) / x\right]$ forsome interpretation $\mathcal{I}$. Since the connectives of $F$ are projective its evaluation only depends on the elements of $\mathcal{M}$ assigned by $\mathcal{I}$ to the propositional variables of $F$ and the constants of $\mathbf{T}$. That is: we can filtrate $\mathcal{M}$ into a model $\mathcal{M}^{\prime}$ with domain $\{\mathcal{I}(p) \mid p$ occurs in $F\} \cup\{c \mid c$ is the value of some constant of $\mathbf{T}\}$. (Remember that the signature of any semantic theory is finite.) Therefore, if $F$ is valid in all finite models it must be valid in arbitrary models, i.e. in $\mathcal{L}_{\mathbf{T}}$.

Observe that the proof provides a bound for the size of models (i.e. number of truth values) that we have to consider if we want to check whether a formula is valid in $\mathcal{L}_{\mathrm{T}}$. In the case of $\mathrm{G}_{\infty}$ we obtain: $F \in \mathrm{G}_{|F|+2}$ implies $F \in \mathrm{G}_{\infty}$ where $|F|$ is the number of (distinct) propositional variables occurring in $F$.

\section{Sequent calculi of relations}

There are quite different ways to interpret Gentzen's classical sequent calculus LK [17]. These lead to different types of generalizations of Gentzen's calculus. One - very useful - interpretation of a sequent

$$
F_{1}, \ldots, F_{n} \longrightarrow G_{1}, \ldots, G_{m}
$$

is to understand it as expressing the assertion that either one of the $F_{i}(1 \leq$ $i \leq n)$ is false or one of the $G_{j}(1 \leq j \leq m)$ is true. In this view a classical sequent can be identified with a sequence

$$
\text { False }\left(F_{1}\right), \ldots, \text { False }\left(F_{n}\right), \operatorname{True}\left(G_{1}\right), \ldots, \operatorname{True}\left(G_{m}\right)
$$

of (monadic) atomic formulas referring to the usual semantic theory. It is well known how this leads to the formulation of sequent calculi for all finite-valued logics (see, e.g., $[23,26,10]$ ).

However, one may prefer to think of the sequent arrow in

$$
F \longrightarrow G
$$

as associated with the binary semantic predicate " $F$ implies $G$ ". In the context of a many-valued logic with an ordered set of truth value this can, e.g., be understood as

$$
\operatorname{val}_{\mathcal{I}}(F) \leq \operatorname{val}_{\mathcal{I}}(G)
$$


for all interpretations $\mathcal{I}$.

The concept of "hypersequents" (as investigated extensively by A. Avron in, e.g., [2,3], see Section 4.1) extends the range of logics for which analytic Gentzen style systems can be given. Hypersequents are sequences of sequents understood as disjunctively connected (at the external level). If external contraction and external weakening are present and a "splitting rule" (which is an instance of Avron's communication rule, introduced in [2] to define a hypersequent calculus for $G_{\infty}$ based on Gentzen's sequent calculus $\mathbf{L J}$ for intuitionistic logic) is admissible, then the hypersequent

$$
\ldots\left|F_{1}, \ldots, F_{n} \longrightarrow G\right| \ldots
$$

is equivalent to the hypersequent

$$
\ldots\left|F_{1} \longrightarrow G\right| \ldots\left|F_{n} \longrightarrow G\right| \ldots
$$

This hypersequent can again be viewed as a sequence of (binary) atomic formulas referring to a semantic theory. (For this one needs truth constants that correspond to an empty left or right hand sight of the sequents.)

The connection to the semantic framework described in Section 2 is manifested in the following definition:

Let $R_{1}, \ldots, R_{n}$ be the predicate symbols of a semantic theory $\mathbf{T}$, then a sequent of relations is a finite multiset ${ }^{3}$ written in form

$$
R_{i_{1}}\left(F_{1}^{1}, \ldots, F_{r_{1}}^{1}\right)|\ldots| R_{i_{k}}\left(F_{1}^{k}, \ldots, F_{r_{k}}^{k}\right)
$$

where for all $1 \leq j \leq k: i_{j} \in\{1, \ldots, n\}, r_{\ell}$ is the arity of $R_{i_{\ell}}$ and all $F_{j}^{i}$ are formulas of a logic. (Strictly speaking, the relational symbols $R_{j}$ just correspond to symbols of the language of $\mathbf{T}$, since the terms of $\mathbf{T}$ are not formulas but variables and constants for truth values.)

We are now going to define the sequent calculus of relations $\mathbf{R} \mathcal{L}_{\mathbf{T}}$ for a projective $\operatorname{logic} \mathcal{L}_{\mathbf{T}}$ defined with respect to a semantic theory $\mathbf{T}$.

\section{Axiom sequents}

Let $\mathbf{T} \models \forall \bar{x} \bigvee_{1 \leq j \leq n} B_{j}$ where the $B_{j}$ are atomic formulas and $\bar{x}$ are the free variables in $\bigvee_{1 \leq j \leq n} B_{j}$. Let $\theta$ be any substitution of formulas for the variables $\bar{x}$. Then

$$
B_{1} \theta|\ldots| B_{n} \theta
$$

is an axiom of $\mathbf{R} \mathcal{L}_{\mathbf{T}}$.

Remark 3. Since $\mathbf{T}$ decides all $\Pi_{1}$-formulas the set of axioms is recursive.

Remark 4. Instead of taking all valid disjunctions of atomic formulas to define axioms one may just consider minimal valid disjunctions. I.e., one reduces the set of axioms modulo the (provability) equivalence relation induced by the structural rules described below.

\footnotetext{
${ }^{3}$ If one prefers sequences over multisets as basic objects of inference then a permutation rule has to be added to the calculus. This approach was used [8].
} 


\section{Structural rules}

As already mentioned above, the structural rules for relational sequents should capture the intended interpretation of "|" as disjunction. Therefore we have the following rules in $\mathbf{R} \mathcal{L}_{\mathbf{T}}$.

$$
\frac{\mathcal{H}}{A \mid \mathcal{H}} \text { weakening } \quad \frac{A|A| \mathcal{H}}{A \mid \mathcal{H}} \text { contraction }
$$

where $A, B$ are arbitrary atomic relations on formulas and $\mathcal{H}$ is an arbitrary (possible empty) side sequent.

We call these rules external since they manipulate whole components (i.e., relations) of sequent but do not change formulas within components.

\section{Logical rules}

Let $\square$ be an $n$-ary projective connective with the following truth function:

$$
\widetilde{\square}\left(x_{1}, \ldots, x_{n}\right)= \begin{cases}t_{1} & \text { if } A_{1}\left(x_{1}, \ldots, x_{n}\right) \\ \vdots & \vdots \\ t_{m} & \text { if } A_{m}\left(x_{1}, \ldots, x_{n}\right)\end{cases}
$$

For each predicate symbol $R$ of arity $r$ and each position $p$, where $1 \leq p \leq r$, we obtain a rule $(\square: R: p)$ for introducing $\square$ at position $p$ into an $R$ component of a relational sequent. For this one considers the formula

$$
\alpha_{\square: R: p}=\bigvee_{1 \leq \ell \leq m} A_{\ell}\left(x_{1}, \ldots, x_{n}\right) \wedge R\left(z_{1}, \ldots, z_{r}\right)\left\{t_{\ell} / z_{p}\right\}
$$

Take any conjunction of disjunctions of atomic formulas $\bigwedge_{1 \leq j \leq s} \bigvee_{1 \leq k \leq u_{j}} B_{j, k}$ that is equivalent in $\mathbf{T}$ to $\alpha_{\square: R: p}$. Then we have the rule

$$
\frac{B_{1,1} \theta|\ldots| B_{1, u_{1}} \theta\left|\mathcal{H} \quad \ldots \quad B_{s, 1} \theta\right| \ldots\left|B_{s, u_{s}} \theta\right| \mathcal{H}}{R\left(z_{1}, \ldots, z_{r}\right)\left\{\square\left(x_{1}, \ldots, x_{n}\right) / z_{p}\right\} \theta \mid \mathcal{H}}(\square: R: p)
$$

where $\theta$ substitutes formulas for the variables $\left\{x_{1}, \ldots, x_{n}\right\} \cup\left\{z_{1}, \ldots, z_{r}\right\}-$ $\left\{z_{p}\right\}$, and $\mathcal{H}$ is the side sequent of the rule.

Remark 5. We make use of the fact that the conditions $A_{i}$ are simple, i.e. negation and quantifier free.

Remark 6. In general there are many conjunctive normal forms equivalent to $\alpha_{\square: R: p}$. To obtain compact rules it is often essential to apply simplifications justified by $\mathbf{T}$-valid formulas.

Remark 7 . The $\alpha_{\square: R: p}$ are $\Pi_{1}$-formulas of $\mathbf{T}$. Since $\mathbf{T}$ decides all $\Pi_{1}$-formulas, the transformation of the specification of a truth function into a logical rule for sequents of relations can - in principle - be automatized. 
Example 2. Continuing Example 1 we arrive at a sequent calculus of (monadic) relations for each finite-valued logic if we follow the above definitions. In fact, because of the presence of the standard structural rules, these calculi are just notational variants of the many-placed sequent calculi or signed calculi as described, e.g., in $[23,10]$. (The special case of classical logic - LK [17] - was already sketched at the beginning of the section.) We can even get rid of the truth constants in the formulation of the calculi. The reason for this is that, obviously, any atomic formula of $\mathbf{T}$ that contains a constant can only be of form $C_{i}\left(c_{j}\right)$, and thus is either simply true or false. For the axioms and rules this means that formulas $C_{i}\left(c_{j}\right)$ where $i \neq j$ are deleted from the sequents, and sequents containing $C_{i}\left(c_{j}\right)$ where $i=j$ are discarded, altogether.

\subsection{Correctness, completeness, decidability}

A sequent (of relations) $\mathcal{S}$ is called provable in $\mathbf{R} \mathcal{L}_{\mathbf{T}}$ if there is an upward tree of sequents rooted in $\mathcal{S}$, such that every leaf (topmost sequent) is an axiom and every other sequent is obtained from the ones standing immediately above it by application of one of the rules of $\mathbf{R} \mathcal{L}_{\mathbf{T}}$.

For any sequent

let

$$
\mathcal{S}=R_{1}\left(F_{1,1}, \ldots, F_{1, r_{1}}\right)|\ldots| R_{n}\left(F_{n, 1}, \ldots, F_{n, r_{n}}\right)
$$

$$
\beta_{\mathcal{S}}=\bigvee_{1 \leq i \leq n} R_{i}\left(t_{i, 1}, \ldots, t_{i, r_{i}}\right)
$$

be the $\mathbf{T}$-formula corresponding to $\mathcal{S}$, where $t_{i, j}$ is identical to $F_{i, j}$ if $F_{i, j}$ is a truth constant ${ }^{4}$ and is a new variable $x_{i, j}$ otherwise $\left(x_{i, j}=x_{k, \ell}\right.$ iff $F_{i, j}=F_{k, \ell}$.

Since the designating predicate Des is a simple formula it is equivalent to a formula $D e s^{\prime}$ of form $\bigwedge_{1 \leq i \leq p} \bigvee_{1 \leq j \leq q_{i}} A_{i, j}$ where the $A_{i, j}$ are atomic formulas with at most one free variable $x$. By

$$
\mathcal{D}_{1}\{x / F\}, \ldots, \mathcal{D}_{p}\{x / F\}
$$

we denote the sequence of sequents that correspond to the conjuncts of $D e s^{\prime}$ if $x$ is replaced by the formula $F$.

For the following statements let $\mathbf{T}$ be any semantic theory and $\mathcal{L}_{\mathbf{T}}$ be the logic determined by $\mathbf{T}$, an object language, projective truth functions for this language and a designating predicate Des. $\mathbf{R} \mathcal{L}_{\mathbf{T}}$ is the corresponding sequent calculus of relations as defined above.

Theorem 1 (Correctness). If all sequents $\mathcal{D}_{1}\{x / F\}, \ldots, \mathcal{D}_{p}\{x / F\}$ are provable in $\mathbf{R} \mathcal{L}_{\mathbf{T}}$ then $F$ is valid in $\mathcal{L}_{\mathbf{T}}$.

\footnotetext{
${ }^{4}$ Remember that we identify the constants of $\mathbf{T}$ with truth constants of the object language.
} 
Proof. We show by induction on the length of proofs that for all models $\mathcal{M}$ of $\mathbf{T}$ and all interpretations $\mathcal{I}: \mathcal{M} \models \beta_{\mathcal{S}}\left[\sigma_{\mathcal{I}}\right]$ if $\mathcal{S}$ is provable, where $\sigma_{\mathcal{I}}$ assigns $\operatorname{val}_{\mathcal{I}}\left(F_{i, j}\right)$ to the corresponding variable $x_{i, j}$. From this the theorem follows by the definition of $\mathcal{D}_{1}\{x / F\}, \ldots, \mathcal{D}_{p}\{x / F\}$ and the fact that $F$ is valid if for all $\mathcal{M}$ and $\mathcal{I}: \mathcal{M} \models \operatorname{Des}\left[\operatorname{val}_{\mathcal{I}}(F) / x\right]$.

For axioms the claim immediately follows from their definition.

For applications of structural rules with premiss $\mathcal{S}$ and conclusion $\mathcal{S}^{\prime}$ we have $\beta_{\mathcal{S}}$ implies $\beta_{\mathcal{S}^{\prime}}$ by the fact that the $\mathbf{T}$-formulas corresponding to sequents are classical disjunctions.

For the application of a logical rule $(\square: R: p)$ it suffices to observe that, by definition, for any $\sigma: \mathcal{M} \models \alpha_{\square: R: p}[\sigma]$ implies that $\mathcal{M} \models R\left(z_{1}, \ldots, z_{r}\right)\left[\sigma^{\prime}\right]$, where $\sigma^{\prime}$ is as $\sigma$ except for assigning $\widetilde{\square}\left(\sigma\left(x_{1}\right), \ldots, \sigma\left(x_{n}\right)\right)$ to the only variable $z_{p}$ that does not already occur in $\alpha_{\square: R: p}$.

Theorem 2 (Completeness). If $F$ is valid in $\mathcal{L}_{\mathbf{T}}$ then all sequents $\mathcal{D}_{1}\{x / F\}$, $\ldots, \mathcal{D}_{p}\{x / F\}$ are provable.

Proof. (Sketch) We employ Schütte's method of reduction trees [24]. That is, we construct a reduction tree $R T$ for every sequent $\mathcal{S}$ such that either a proof of $\mathcal{S}$ or a model in which $\beta_{\mathcal{S}}$ is not valid can be extracted from $R T$.

The construction of the upward tree of sequents $R T$ for the sequent $\mathcal{S}$ is in stages as follows:

Stage 0: Write $\mathcal{S}$ at the root of $R T$.

Stage $k$ : If the topmost sequent $\mathcal{S}^{\prime}$ of a branch contains only propositional variables (as arguments of its relations) then stop the reduction for this branch. Otherwise $\mathcal{S}^{\prime}$ contains a relation $R\left(F_{1}, \ldots, F_{r}\right)$ where $F_{p} \equiv \square\left(G_{1}\right.$, $\left.\ldots, G_{n}\right)$ for some $1 \leq p \leq r$. If the indicated occurrence of $\square\left(G_{1}, \ldots, G_{n}\right)$ is not the result of a reduction at this stage and has not yet been reduced on this branch then replace $\mathcal{S}^{\prime}$ by

$$
\frac{B_{1,1} \theta|\ldots| B_{1, t_{1}} \theta\left|\mathcal{S}^{\prime} \quad \ldots \quad B_{s, 1} \theta\right| \ldots\left|B_{s, t_{s}} \theta\right| \mathcal{S}^{\prime}}{\mathcal{S}^{\prime}}
$$

where the $B_{i, j}$ are as in the definition of rule $(\square: R: p)$ and $\theta$ is given by $R\left(z_{1}, \ldots, z_{r}\right)\left\{\square\left(x_{1}, \ldots, x_{n}\right) / z_{p}\right\} \theta=R\left(F_{1}, \ldots, F_{r}\right)$.

Since every occurrence of a formula is only reduced once in a branch, the construction of $R T$ stops after finitely many steps.

We say that a sequent $\mathcal{S}^{\prime}$ contains an axiom if it can be derived from an axiom using structural rules only. If each leaf of $R T$ contains an axiom of $\mathbf{R} \mathcal{L}_{\mathbf{T}}$ then a proof of $\mathcal{S}$ is easily constructed from $R T$ by inserting weakenings and contractions.

Otherwise there is a leaf sequent $\mathcal{R}$ that does not contain an axiom. Let $\beta_{\mathcal{R}}$ be the $\mathbf{T}$-formula corresponding to $\mathcal{R}$ (by replacing the propositional variables $f_{i}$ occurring in $\mathcal{R}$ by variables $x_{i}$ of the language of $\mathbf{T}$ ). 
By definition of the set of axioms, there is a model $\mathcal{M}$ of $\mathbf{T}$ and an assignment $\sigma$ such that $\mathcal{M} \not \forall \beta_{\mathcal{R}}[\sigma]$. The assignment $\sigma$ of truth values to the $x_{i}$ induces an interpretation $\mathcal{I}$ of the corresponding propositional variables $f_{i}$. By going down the branch from $\mathcal{R}$ to the root $\mathcal{S}$ one can augment $\mathcal{I}$ to an interpretation of all propositional variables occurring in $\mathcal{S}$ such that $\mathcal{M} \not \models \beta_{\mathcal{S}}\left[\operatorname{val}_{\mathcal{I}}\left(H_{1}\right) / x_{1}, \ldots, \operatorname{val}_{\mathcal{I}}\left(H_{k}\right) / x_{k}\right]$, where the $H_{i}$ are the formulas in $\mathcal{S}$. (For this, of course, one has to use the corresponding truth functions as interpreted in $\mathcal{M}$.)

$F$ is valid in $\mathcal{L}_{\mathbf{T}}$ iff for all $\mathcal{M}$ and $\mathcal{I}, \mathcal{M}=\forall x \operatorname{Des}\left[\operatorname{val}_{\mathcal{I}}(F) / x\right]$. Since $\mathbf{T} \models \forall x$ Des $\Leftrightarrow \bigwedge_{1<i<p} \mathcal{D}_{i}$ it follows that all leaves of a reduction tree $R T_{i}$ for a sequent $\mathcal{D}_{i}\{x / F\}$ contain axioms. Therefore all sequents $\mathcal{D}_{1}\{x / F\}, \ldots$, $\mathcal{D}_{p}\{x / F\}$ are provable in $\mathbf{R} \mathcal{L}_{\mathbf{T}}$ if $F$ is valid.

Since the construction of the reduction trees is effective we obtain:

Corollary 1. All projective logics $\mathcal{L}_{\mathbf{T}}$ are decidable.

Remark 8. The construction of the reduction tree can be seen as the search for a proof in tableau format. Here, the atomic elements of the tableau are not just formulas but (atomic) relations between formulas. The reduction of compound formulas corresponds to the introduction rules of the sequent calculus. The tableau closure rules correspond to the axioms. The close relationship between reduction trees (i.e., tableaux) and sequent proofs relies on the fact that, like in classical logic, we can view sequents as sets (i.e., modulo contraction) and can move all weakenings up to the axioms.

\subsection{Extended structural rules}

So far we only considered (analytic) rules for introducing connectives and traditional forms of structural rules. Let us now investigate

which types of cut rules or more general forms of structural rules

(that possibly allow to exchange formulas from different relations in sequents) are admissible in our calculi. Although we know - by completeness - that such rules are not needed for proof search, one should keep in mind that vast speedups (at least with respect to proof length) can be gained by applying such rules. This is already well known for the "simplest" case of a projective logic, namely classical logic.

We call a rule extended structural rule if it is of the form:

$$
\begin{array}{ccc}
\mathcal{H} \mid \Gamma_{1} \theta & \ldots & \mathcal{H} \mid \Gamma_{n} \theta \\
\mathcal{H} \mid \Gamma \theta
\end{array}
$$

where $\Gamma_{1}, \ldots, \Gamma_{n}, \Gamma$ are sequences of atomic formulas of $\mathbf{T}$ (separated by "|"), $\theta$ is a substitution of variables by formulas and $\mathcal{H}$ a side sequent. 
Remark 9. Because of the presence of weakening and contraction, there is no loss of generality in considering only identical side sequents in the premisses. Indeed this "additive" version of rules (see [28]) is more suitable in the context of tableau style proof search.

An extended structural rule is admissible in $\mathbf{R} \mathcal{L}_{\mathbf{T}}$ if

$$
\mathbf{T} \models \forall \bar{x}\left(\widehat{\Gamma_{1}} \wedge \ldots \wedge \widehat{\Gamma_{n}}\right) \supset \widehat{\Gamma}
$$

where $\bar{x}$ is the vector of all variables occurring in $\Gamma_{1}, \ldots, \Gamma_{n}, \Gamma$ and $\widehat{\Delta}$ is the disjunction of the atomic formulas $\Delta$ consists of. ( $\widehat{\Delta} \equiv$ True for empty $\Delta$.)

It follows from this definition that, indeed, all sequents provable in $\mathbf{R} \mathcal{L}_{\mathbf{T}}$ augmented by admissible extended structural rules are already provable in $\mathbf{R} \mathcal{L}_{\mathbf{T}}$ without these rules.

It is important to notice that admissibility is a decidable property of rules, because we required all $\Pi_{1}$-sentences to be decidable in $\mathbf{T}$.

Let $\operatorname{var} s(\Delta)$ denote the set of variables occurring in the sequent $\Delta$. We call an extended structural rule cut rule if $\operatorname{vars}(\Gamma) \subsetneq \bigcup_{1 \leq i \leq n} \operatorname{var} s\left(\Gamma_{i}\right)$. (That is at least one formula is "cut out" from the premisses.) If $\operatorname{var} s(\Gamma) \supseteq$ $\bigcup_{1 \leq i \leq n} \operatorname{vars}\left(\Gamma_{i}\right)$ we speak of an analytic structural rule.

Remark 10. In general, many different extended structural rules are admissible. They constitute an open list of possible (i.e., admissible) but (for completeness) not necessary extensions of the analytic calculi defined in Section 3. Some examples are as follows:

Example 3. If $\mathbf{T}$ contains a transitive relation " $\prec$ " — e.g., " $<$ " and " $\leq$ " in the semantic theory $\mathbf{T}_{\leq,<}$of Gödel logics — then

$$
\frac{F \prec G|\mathcal{H} \quad G \prec H| \mathcal{H}}{F \prec H \mid \mathcal{H}} \text { (tr-cut) }
$$

is an admissible cut rule, called transitivity-cut.

If the partial ordering $\prec$ has a minimal element 0 and a maximal element 1 distinct from 0 - again Gödel logics are concrete examples — then

$$
\frac{F \prec 0|\mathcal{H} \quad 1 \prec F| \mathcal{H}}{\mathcal{H}}
$$

is another admissible cut rule.

If $\prec$ is irreflexive — as " $<$ " for Gödel logics — also the unary cut rules

$$
\frac{1 \prec F \mid \mathcal{H}}{\mathcal{H}} \quad \text { and } \quad \frac{F \prec 0 \mid \mathcal{H}}{\mathcal{H}}
$$

are admissible. 
Example 4. Let " $\leq$ " and 0,1 be as in the semantic theory $\mathbf{T}_{\leq,<}$for Gödel logics. Then

$$
\frac{1 \leq F \mid \mathcal{H}}{G \leq F \mid \mathcal{H}}(w: \leq: l) \quad, \quad \frac{G \leq 0 \mid \mathcal{H}}{G \leq F \mid \mathcal{H}}(w: \leq: r) \quad \text { and } \quad \frac{1 \leq 0 \mid \mathcal{H}}{\mathcal{H}}
$$

are examples of admissible analytic structural rules. The first two correspond to (internal) weakening in standard sequent calculi.

An important analytic structural rule, admissible for Gödel logics, is:

$$
\frac{F \leq G|\mathcal{H} \quad H \leq I| \mathcal{H}}{H \leq G|F \leq I| \mathcal{H}}
$$

It corresponds to an instance of Avron's communication rule as we shall see in Section 4.1

\section{A sequent of relations calculus for Gödel logics}

As an illustration of the proof theoretic framework of the last section we present sequent of relations calculi for the sequent of relations calculus $\mathbf{R G}_{\infty}$ for infinite-valued Gödel logic $\mathrm{G}_{\infty}$. Calculi for $G_{n}$ and for $\mathrm{G}_{\infty}^{\Delta}$ are obtained from $\mathbf{R G}_{\infty}$ by adding suitable axioms or rules, as explained in Remarks 11 and 12 , respectively.

The rules of $\mathbf{R} \mathbf{G}_{\infty}$ are derived using the semantic theory $\mathbf{T}_{\leq,<}$(see Section 2.1) as described in Section 3.

Remember, that the (external) structural rules are the same for all sequent of relations calculi. For $\mathbf{R} \mathbf{G}_{\infty}$ they can be written as follows:

$$
\frac{\mathcal{H}}{A \triangleleft B \mid \mathcal{H}} \text { weakening } \quad \frac{A \triangleleft B|A \triangleleft B| \mathcal{H}}{A \triangleleft B \mid \mathcal{H}} \text { contraction }
$$

where $\triangleleft \in\{<, \leq\}$.

The axioms of $\mathbf{R G}_{\infty}$ are all sequents that contain a sequent

$$
A_{1} \triangleleft_{1} A_{2}\left|A_{2} \triangleleft_{2} A_{3}\right| \ldots \mid A_{k} \triangleleft_{k} A_{1}
$$

for $k \geq 1$, where $\triangleleft_{i} \in\{<, \leq\}$ for all $1 \leq i \leq k$, but $\triangleleft_{i} \equiv \leq$ for at least one $i$. In addition, all sequents that are obtained from the above ones by deleting relations of form

$$
A<0, \quad 1<A, \quad \text { or } \quad 1 \leq 0
$$

are axioms.

In [7] the following explicit description of the above axioms has been introduced:

(a) $A_{1} \triangleleft_{n} A_{n}|\ldots| A_{3} \triangleleft_{2} A_{2} \mid A_{2} \leq A_{1}$, where $\triangleleft_{i} \in\{<, \leq\}$ and the case $n=1$ is defined as $A_{1} \leq A_{1}$, 
(b) $A_{n} \leq A_{n-1}\left|A_{n-1}<A_{n-2}\right| \ldots \mid A_{1}<1$, where the case $n=1$ is defined as $A_{1} \leq 1$,

(c) $0<A_{n}|\ldots| A_{3}<A_{2} \mid A_{2} \leq A_{1}$, where the case $n=1$ is defined as $0 \leq A_{1}$

(d) $0<A_{1}\left|A_{1}<A_{2}\right| \ldots \mid A_{n}<1$, where the case $n=0$ is defined as $0<1$.

The above description, corresponds to the original set of axioms up to (external) weakening.

Theorem 3. Axioms of type $(a)-(d)$ are, up to (external) weakening, all and only the atomic sequents valid in $\mathbf{T}_{\leq,<}$.

It is easy to check that all of the above axioms correspond to valid statements in $\mathbf{T}_{\leq,<}$. However, to guarantee completeness we also have to show the converse: namely, that all valid atomic sequents are obtained from these axioms using external weakening only. For this purpose it is better to consider the dual form of the axioms. I.e., we make use of the fact that $v a l_{\mathcal{I}}(A)<v a l_{\mathcal{I}}(B)$ iff $\neg\left[\operatorname{val}_{\mathcal{I}}(B) \leq \operatorname{val}_{\mathcal{I}}(A)\right]$, and thus may consider conjunctions of components instead of disjunctions.

Definition 1. A set of components is called dual to axioms if it does not contain any subset of one of the following forms:

(a) (anti-cycle) $\left\{A_{1}<A_{2}, A_{2} \triangleleft_{2} A_{3}, \ldots, A_{n} \triangleleft_{n} A_{1}\right\}$, where $\triangleleft_{i} \in\{<, \leq\}$ and the case $n=1$ is defined as $\left\{A_{1}<A_{1}\right\}$,

(b) (anti-1-chain) $\left\{1 \leq A_{1}, \ldots, A_{n-2} \leq A_{n-1}, A_{n-1}<A_{n}\right\}$, where the case $n=1$ is defined as $\left\{1<A_{1}\right\}$,

(c) (anti-0-chain) $\left\{A_{1}<A_{2}, A_{2} \leq A_{3}, \ldots, A_{n} \leq 0\right\}$, where the case $n=1$ is defined as $\left\{A_{1}<0\right\}$,

(d) (anti-0-1-chain) $\left\{1 \leq A_{1}, A_{2} \leq A_{3}, \ldots, A_{n} \leq 0\right\}$, where the case $n=0$ is defined as $\{1 \leq 0\}$.

It suffices to prove the following:

Theorem 4. Let $\Gamma$ be a finite set of components $A \triangleleft B, \triangleleft \in\{<, \leq\}$, where $A$ and $B$ are either propositional variables or truth constants. If $\Gamma$ is dual to axioms then $\Gamma$ is satisfiable; i.e., there exists an interpretation that satisfies all components of $\Gamma$.

To prove Theorem 4 we extend any $\Gamma$ that is dual to axioms to a "maximal" set $\Gamma^{*}$ that is still dual to axioms. Let us write $B \in[A] \Longleftrightarrow\{A \leq$ $B, B \leq A\} \subseteq \Gamma^{*}$. It will follow from Proposition 2 and Lemma 1, below, that this is an equivalence relation and that the set of equivalence classes $\overline{\Gamma^{*}}=\left\{[A]: A\right.$ occurs in $\left.\Gamma^{*}\right\}$ is totally ordered with respect to $[A]<[B] \Longleftrightarrow$ $A<B \in \Gamma^{*}$. The minimal element of the ordering is [0] and its maximal element is [1] (if 0 and 1 occur in $\Gamma$ ). The ordering thus allows to match equivalence classes with truth values in a way that induces an interpretation satisfying $\Gamma^{*}$ and therefore also $\Gamma$.

We first add $A \leq B$ to $\Gamma$ whenever $A<B \in \Gamma$. This is justified by the following simple observation: 
Proposition 2. If $\Gamma$ is dual to axioms then $\Gamma \cup\{A \leq B: A<B \in \Gamma\}$ is dual to axioms, too.

The existence of $\overline{\Gamma^{*}}$ follows from the following:

Lemma 1. If $\Gamma$ is dual to axioms then either $\Gamma \cup\{A<B\}$ or $\Gamma \cup\{B \leq A\}$ is dual to axioms, too.

Proof. The proof proceeds by case distinctions:

(1) $\Gamma \cup\{A<B\}$ contains an anti-cycle. Then either already $\Gamma$ contains an anti-cycle or $\left\{B \leq U_{1}, \ldots, U_{n} \leq A\right\} \subseteq \Gamma$. From this it follows that $\Gamma \cup\{B \leq A\}$ is dual to axioms iff $\Gamma$ is dual to axioms.

(2) $\Gamma \cup\{B \leq A\}$ contains an anti-cycle. Then either already $\Gamma$ contains an anti-cycle or $\left\{A \leq U_{1}, \ldots, U_{k}<U_{k+1}, \ldots U_{n} \leq B\right\} \subseteq \Gamma$. From this it follows that $\Gamma \cup\{A<B\}$ is dual to axioms iff $\Gamma$ is dual to axioms.

(3) Neither $\Gamma \cup\{A<B\}$ nor $\Gamma \cup\{B \leq A\}$ contains an anti-cycle.

(3.1) $\Gamma \cup\{A<B\}$ contains an anti-1-chain W.l.o.g., the anti-1-chain is not already contained in $\Gamma$. Therefore (a): $\left\{1 \leq V_{1}, \ldots, V_{n-1} \leq A\right\} \subseteq \Gamma$.

(3.1.1) $\Gamma \cup\{B \leq A\}$ contains an anti-1-chain that is not already contained in $\Gamma$. Therefore $\left\{1 \leq U_{1}, \ldots, U_{k-1} \leq B\right\} \subseteq \Gamma$ and $\{A \leq$ $\left.U_{k+1}, \ldots, U_{k+m-1}<U_{k+m}\right\} \subseteq \Gamma$. The latter subset can be combined with (a) to an anti-1-chain in $\Gamma$.

(3.1.2) $\Gamma \cup\{B \leq A\}$ contains an anti-0-chain that is not already contained in $\Gamma$. Therefore $\left\{U_{1}<U_{2}, \ldots, U_{k-1} \leq B\right\} \subseteq \Gamma$ and $\left\{A \leq U_{k+1}, \ldots, U_{k+m} \leq 0\right\} \subseteq \Gamma$. The latter subset can be combined with (a) to an anti-0-1-chain in $\Gamma$.

(3.1.3) $\Gamma \cup\{B \leq A\}$ contains an anti-0-1-chain that is not already contained in $\Gamma$. Therefore $\left\{1 \leq U_{1}, \ldots, U_{k-1} \leq B\right\} \subseteq \Gamma$ and $\left\{A \leq U_{k+1}, \ldots, U_{k+m} \leq 0\right\} \subseteq \Gamma$. The latter subset can be combined with (a) to an anti-0-1-chain in $\Gamma$.

(3.2) $\Gamma \cup\{B \leq A\}$ contains an anti-1-chain that is not already contained in $\Gamma$. Therefore

(b1): $\left\{1 \leq V_{1}, \ldots, V_{k-1} \leq B\right\} \subseteq \Gamma$ and (b2): $\left\{A \leq V_{k+1}, \ldots\right.$, $\left.V_{k+m-1}<V_{k+m}\right\} \subseteq \Gamma$.

(3.2.1) $\Gamma \cup\{A<B\}$ contains an anti-1-chain. This case was already settled in (3.1.1).

(3.2.2) $\Gamma \cup\{A<B\}$ contains an anti-0-chain that is not already contained in $\Gamma$. Then $\left\{B \leq U_{2}, \ldots, U_{n} \leq 0\right\} \subseteq \Gamma$. This subset can be combined with (b1) to an anti-0-1-chain in $\Gamma$.

(3.3) Neither $\Gamma \cup\{A<B\}$ nor $\Gamma \cup\{A \leq B\}$ contain an anti-1-chain.

(3.3.1) $\Gamma \cup\{A<B\}$ contains an anti-0-chain that is not already contained in $\Gamma$. Then (c) $\left\{B \leq V_{2}, \ldots, V_{n} \leq 0\right\} \subseteq \Gamma$.

(3.3.1.1) $\Gamma \cup\{B \leq A\}$ contains an anti-0-chain that is not already contained in $\Gamma$. Therefore $\left\{U_{1}<U_{2}, \ldots, U_{k-1} \leq B\right\} \subseteq \Gamma$ and $\left\{A \leq U_{k+1}, \ldots, U_{k+m} \leq 0\right\} \subseteq \Gamma$. The first subset can be combined with (c) to an anti-0-chain in $\Gamma$. 
(3.3.1.2) $\Gamma \cup\{B \leq A\}$ contains an anti-0-1-chain that is not already contained in $\Gamma$. Therefore $\left\{1<U_{2}, \ldots, U_{k-1} \leq B\right\} \subseteq \Gamma$ and $\left\{A \leq U_{k+1}, \ldots, U_{k+m} \leq 0\right\} \subseteq \Gamma$. The first subset can be combined with (c) to an anti-0-1-chain in $\Gamma$.

Finally observe that if $\Gamma \cup\{A<B\}$ contains an anti-0-1-chain then this anti-0-1-chain is already contained in $\Gamma$. It is easy to check that this settles all remaining cases.

The logical rules of $\mathbf{R} \mathrm{G}_{\infty}$ - i.e., the rules for introducing connectives at any place of a sequent - are easily derived as described in Section 3 above by manipulating the rule-defining formulas $\alpha_{\square: R: p}$ in $\mathbf{T}_{\leq,<}$. Here $R \in\{<, \leq\}$; $p \in\{l, r\}$ for the left and right argument position of the binary relations, respectively, and $\square \in\{\supset, \wedge, \vee\}$.

$$
\begin{aligned}
\alpha_{(\supset:<: r)} \equiv & (x \leq y \wedge z<1) \vee(y<x \wedge z<y) \\
\Longleftrightarrow & (x \leq y \vee y<x) \wedge(x \leq y \vee z<y) \wedge \\
& (z<1 \vee y<x) \wedge(z<1 \vee z<y) \\
\Longleftrightarrow & (x \leq y \vee z<y) \wedge(z<1)
\end{aligned}
$$

The rule for introducing implication at the right argument place of " $<$ " in a relational sequent can therefore be stated as:

$$
\frac{A \leq B|C<B| \mathcal{H} \quad C<1 \mid \mathcal{H}}{C<(A \supset B) \mid \mathcal{H}}(\supset:<: r)
$$

Similarly we have in $\mathbf{T}_{\leq,<}$

$$
\begin{aligned}
\alpha_{(\supset:<: l)} & \equiv(x \leq y \wedge 1<z) \vee(y<x \wedge y<z) \\
& \Longleftrightarrow y<x \wedge y<z
\end{aligned}
$$

Thus a rule for introducing implication at the left argument place of " $<$ " is:

$$
\frac{B<A|\mathcal{H} \quad B<C| \mathcal{H}}{(A \supset B)<C \mid \mathcal{H}}(\supset:<: l)
$$

For implication at the right hand side of the <-relations we obtain:

$$
\begin{aligned}
\alpha_{(\supset: \leq: r)} & \equiv(x \leq y \wedge z \leq 1) \vee(y<x \wedge z \leq y) \\
& \Longleftrightarrow(x \leq y \vee y<x) \wedge(x \leq y \vee z \leq y) \\
& \Longleftrightarrow x \leq y \vee z \leq y
\end{aligned}
$$

This induces the rule

$$
\frac{A \leq B|C \leq B| \mathcal{H}}{C \leq(A \supset B) \mid \mathcal{H}}(\supset: \leq: r)
$$


A compact rule for introducing implication at the left hand side of the $\leq-$ relations is obtained by the following derivation in $\mathbf{T}_{\leq,<}$:

$$
\begin{aligned}
\alpha_{(\supset: \leq: l)} \equiv & (x \leq y \wedge 1 \leq z) \vee(y<x \wedge y \leq z) \\
\Longleftrightarrow & (x \leq y \vee y<x) \wedge(x \leq y \vee y \leq z) \wedge \\
& (1 \leq z \vee y<x) \wedge(1 \leq z \vee y \leq z) \\
\Longleftrightarrow & (1 \leq z \vee y<x) \wedge(y \leq z)
\end{aligned}
$$

This induces the rule

$$
\frac{1 \leq C|B<A| \mathcal{H} \quad B \leq C \mid \mathcal{H}}{(A \supset B) \leq C \mid \mathcal{H}}(\supset: \leq: l)
$$

Observe that this is the only $\leq$-rule exhibiting " $<$ " in the premisses. (This is of importance for the connection to Avron's hypersequent calculus $\mathbf{G} L C$ for $\mathrm{G}_{\infty}$; see Section 4.1 below.)

Computing the rules for disjunction and conjunction is easy. They take the same form for both relations. We therefore let $\triangleleft$ stand for either $<$ or $\leq$ (uniformly in each rule):

$$
\begin{aligned}
& \frac{C \triangleleft A|\mathcal{H} \quad C \triangleleft B| \mathcal{H}}{C \triangleleft(A \wedge B) \mid \mathcal{H}}(\wedge: \triangleleft: r) \quad \frac{A \triangleleft C|B \triangleleft C| \mathcal{H}}{(A \wedge B) \triangleleft C \mid \mathcal{H}}(\wedge: \triangleleft: l) \\
& \frac{C \triangleleft A|C \triangleleft B| \mathcal{H}}{C \triangleleft(A \vee B) \mid \mathcal{H}}(\vee: \triangleleft: r) \quad \frac{A \triangleleft C|\mathcal{H} \quad B \triangleleft C| \mathcal{H}}{(A \vee B) \triangleleft C \mid \mathcal{H}}(\vee: \triangleleft: l)
\end{aligned}
$$

Remark 11. To obtain a calculus for $n$-valued Gödel logic $\mathrm{G}_{n}$ one has to add to $\mathbf{R G}_{\infty}$ all axioms of form

$$
A_{1} \triangleleft_{1} A_{2}\left|A_{2} \triangleleft_{2} A_{3}\right| \ldots \mid A_{l} \triangleleft_{l} A_{l+1}
$$

where $\triangleleft_{i} \equiv \leq$ for at least $n$ different $i \in\{1, \ldots, l\}$, where $l>n$. Below, we refer to this calculus as $\mathbf{R G}_{n}$.

Remark 12. In [6] $\mathrm{G}_{\infty}$ is extended with the "projection modality" $\Delta$. In the resulting logic $\mathrm{G}_{\infty}^{\Delta}$, one can make "fuzzy" statements "crisp", since a formula $\Delta P$ is mapped to the distinguished truth value 1 if the value of $P$ equals 1 , and to 0 otherwise. Clearly, $\Delta$ is projective with respect to the semantic theory $\mathbf{T}_{\leq,<}$of Section 2.1. Indeed, its truth function can be defined as:

$$
\tilde{\Delta}(x)= \begin{cases}1 & \text { if } 1 \leq x \\ 0 & \text { if } x<1\end{cases}
$$

To obtain a calculus for $\mathrm{G}_{\infty}^{\Delta}$ one has to add to $\mathbf{R G}_{\infty}$ suitable rules for introducing $\Delta$. These rules, defined using the algorithm described in Section 3, are as follows:

$$
\frac{A<1 \mid 1 \leq B}{\Delta A \leq B}(\Delta: \leq: l) \quad \frac{B \leq 0 \mid 1 \leq A}{B \leq \Delta A}(\Delta: \leq: r)
$$




$$
\frac{0<B \quad A<1}{\Delta A<B}(\Delta:<: l) \quad \frac{B<1 \quad 1 \leq A}{B<\Delta A}(\Delta:<: r)
$$

Remark 13. As pointed out in Section 3.2, different forms of cuts are admissible in $\mathbf{R G}_{\infty}$. In [7] the following version of the cut rule has been considered

$$
\frac{\mathcal{H}|A \leq B \quad \mathcal{H}| B<A}{\mathcal{H}}\left(\operatorname{cut}_{</ \geq}\right)
$$

and a constructive proof for the elimination of $\left(\mathrm{cut}_{</ \geq}\right)$from given derivations has been presented.

Focusing on $\left(\right.$ cut $\left._{</ \geq}\right)$is motivated by the fact that it allows to simulate other forms of cut straightforwardly. E.g., the transitivity-cut (see Section $3.2)$

$$
\frac{A \leq B|\mathcal{H} \quad B \leq C| \mathcal{H}}{A \leq C \mid \mathcal{H}}\left(\text { tr-cut }_{\leq}\right)
$$

can be derived from a 3-component-cycle by applying $\left(\right.$ cut $\left._{</ \geq}\right)$twice in the following way:

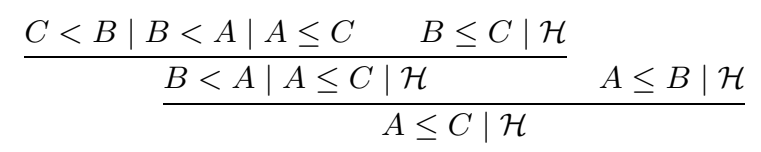

Similar admissible rules involving $<$ instead of $\leq$ can be treated analogously.

As we shall see in Section 4.1 below, $\left(\operatorname{cut}_{</ \geq}\right)$also allows to derive a version of Avron's communication rule.

A different type of admissible rule, related to cut, is the so-called TakeutiTitani rule. This rule, which expresses the density of the set of truth values, has been used in [27] to axiomatize first-order $\mathrm{G}_{\infty}$ :

$$
\frac{F \leq p|p \leq G| \mathcal{H}}{F \leq G \mid \mathcal{H}}(\mathrm{tt})
$$

where $p$ is a propositional variable not occurring in the lower sequent. It is interesting to observe that $(t t)$ cannot be derived in $\mathbf{R G}_{\infty}$ since - in contrast to $\left(\right.$ cut $_{</ \geq}$) and the other rules of $\mathbf{R G}_{\infty}-(t t)$ is not strongly sound, e.g., in finite-valued Gödel logics.

\subsection{Relationships with Hypersequent Calculi and Decomposition Proof Systems for Gödel logics}

We first relate our approach to hypersequent calculi for Gödel logics.

As already mentioned in Section 3, a hypersequent is a finite sequence of sequents (see [3] for an overview). In [2] Avron introduced a hypersequent calculus for $\mathrm{G}_{\infty}$. This calculus - called $\mathbf{G} L C$ - is defined by embedding Gentzen's LJ-sequents into hypersequents. Suitable structural rules are 
added to manipulate the additional layer of structure to the basic objects of inferences. More precisely, these structural rules are external permutation, external weakening, external contraction and the communication rule:

$$
\frac{\Gamma, \Gamma^{\prime} \Rightarrow U\left|\mathcal{H} \quad \Gamma_{1}, \Gamma_{1}^{\prime} \Rightarrow V\right| \mathcal{H}}{\Gamma, \Gamma_{1} \Rightarrow U\left|\Gamma^{\prime}, \Gamma_{1}^{\prime} \Rightarrow V\right| \mathcal{H}}(\mathrm{com})
$$

This rule increases the expressive power of $\mathbf{G} L C$ compared to $\mathbf{L J}$ but is problematic from a computational point of view.

$\mathbf{G} L C$ can be viewed as a calculus of relations. For this purpose we use an equivalent formulation, where the hypersequents are fully split (see Section 3). This version of Avron's calculus, consequently, consists of:

- axioms $A \leq A$

- (external) structural rules

- internal weakening rules $(w: \leq: l)$ and $(w: \leq: r)$ (see Example 4)

- all $\leq$-rules of $\mathbf{R G}_{\infty}$ with the exception of $(\supset: \leq: l)$, which is replaced by

$$
\frac{D \leq A|\mathcal{H} \quad B \leq C| \mathcal{H}}{(A \supset B) \leq C|D \leq C| \mathcal{H}}(\supset: \leq: l)^{*}
$$

- the communication rule:

$$
\frac{A_{1} \leq U|\ldots| A_{n} \leq U\left|\mathcal{H} \quad B_{1} \leq V\right| \ldots\left|B_{m} \leq V\right| \mathcal{H}}{A_{1} \leq V|\ldots| A_{n} \leq V\left|B_{1} \leq U\right| \ldots\left|B_{m} \leq U\right| \mathcal{H}}(\text { rcom) }
$$

It is not hard to see that $\mathbf{R} \mathrm{G}_{\infty}$ and (the above version of) $\mathbf{G} L C$ are equivalent. Indeed, $(\supset: \leq: l)^{*}$ is derivable from $(\supset: \leq: l)$ in $\mathbf{R G}_{\infty}$ using transitivitycuts which can be eliminated from proofs (see Remark 13 and [7]):

$$
\begin{aligned}
& \frac{D \leq A|\mathcal{H} \quad A \leq B| B<A}{\frac{D \leq B|B<A| \mathcal{H}}{D \leq C|B<A| \mathcal{H}} \text { (tr-cut) } \quad B \leq C \mid \mathcal{H}} \text { (tr-cut) } \\
& \frac{\frac{D \leq C|B<A| \mathcal{H}}{1 \leq C|B<A| D \leq C \mid \mathcal{H}} \text { (weak.) } \quad B \leq C \mid \mathcal{H}}{A \supset B \leq C|D \leq C| \mathcal{H}}(\supset: \leq: l)
\end{aligned}
$$

Moreover $(\mathrm{rcom})$ is derivable in $\mathbf{R} \mathrm{G}_{\infty}$ using 4-component-cycles. E.g., in (rcom), let $n=m=1$, one has:

$$
\frac{B_{1} \leq V \mid \mathcal{H} \quad \frac{A_{1} \leq U\left|\mathcal{H} \quad U<A_{1}\right| A_{1} \leq V\left|V<B_{1}\right| B_{1} \leq U}{A_{1} \leq V\left|V<B_{1}\right| B_{1} \leq U \mid \mathcal{H}}}{A_{1} \leq V\left|B_{1} \leq U\right| \mathcal{H}}
$$

Consequently, $\mathbf{R G}_{\infty}$ simulates Avron's $\mathbf{G} L C$.

In the other direction, it easy to show that pure $\leq$-sequents derivable in $\mathbf{R} \mathrm{G}_{\infty}$ are also derivable in (the above version of) $\mathbf{G} L C$ : Let $(A<B)^{d} \equiv$ $1 \leq((B \supset A) \supset B)$ and $(A \leq B)^{d} \equiv 1 \leq A \supset B$. Then all ${ }^{d}$-translations of derivable $\mathbf{R G}_{\infty}$-sequents are derivable in $\mathbf{G} L C$, since the translations of $\mathbf{R} \mathrm{G}_{\infty}$-rules are derivable in $\mathbf{G} L C$. 
Concerning efficient proof search, the most important feature of $\mathbf{R} \mathbf{G}_{\infty}$ is the fact that all the rules are invertible. In particular, we do not have to use the communication rule (or any similar rule destroying the "locality" of tableau style proof search). On the other hand, even if we enrich $\mathbf{G} L C$ by the (more general) axioms of $\mathbf{R G}_{\infty}$ the valid sequent

$$
A \supset B \leq C|A \leq B| C \leq B
$$

is not cut-free provable without using the communication rule. In other words: the communication rule cannot be avoided if " $<$ " is eliminated from the signature. This is connected with the fact that this rule cannot be permuted over the rule for introducing $\supset$ on the left while can be permuted over all the remaining rules of $\mathbf{G} L C$.

Remark 14. A hypersequent calculus $\mathbf{G} L C_{n}$ for $n$-valued Gödel logic, with $n \geq 2$, has been introduced in [13]. This calculus is obtained by replacing, in $\mathbf{G} L C$, the communication rule with the following one:

$$
\frac{\Gamma_{1}, \Gamma_{2} \Rightarrow U_{1}\left|\mathcal{H} \quad \Gamma_{2}, \Gamma_{3} \Rightarrow U_{2}\right| \mathcal{H} \quad \ldots \quad \Gamma_{n-1}, \Gamma_{n} \Rightarrow U_{n-1} \mid \mathcal{H}}{\left(G_{n}\right)}
$$

In analogy to the case above, it is not hard to see that the sequent of relations calculus $\mathbf{R} G_{n}$ (see Remark 11) simulates $\mathbf{G} L C_{n}$ (and vice versa). As an example consider the following (sequent of relations) version of the $\left(G_{3}\right)$ rule

$$
\frac{A_{1} \leq U_{1}\left|A_{2} \leq U_{1}\right| \mathcal{H} \quad A_{2} \leq U_{2}\left|A_{3} \leq U_{2}\right| \mathcal{H}}{A_{1} \leq U_{1}\left|A_{2} \leq U_{2}\right| A_{3} \leq 0 \mid \mathcal{H}}\left(G_{3}\right)
$$

which can be derived in $\mathbf{R G}_{3}$ as follows:

$$
\frac{A_{1} \leq U_{1}\left|A_{2} \leq U_{1} \quad A_{1} \leq U_{1}\right| U_{1}<A_{2}\left|A_{2} \leq U_{2}\right| U_{2}<A_{3} \mid A_{3} \leq 0}{\leq U_{2} \mid A_{3} \leq U_{2}} \frac{A_{1} \leq U_{1}\left|A_{2} \leq U_{2}\right| U_{2}<A_{3} \mid A_{3} \leq 0}{A_{1} \leq U_{1}\left|A_{2} \leq U_{2}\right| A_{3} \leq 0} }
$$

$\mathbf{R G}_{\infty}$ is also closely related with the decomposition proof system $\mathrm{GLC}_{R S}^{*}$ introduced in [5]. This calculus has been defined following the general RasiowaSirkorski methodology for constructing analytic proof systems for semantically defined logics. $\mathrm{GLC}_{R S}^{*}$ and $\mathbf{R G}_{\infty}$ are interderivable using simple notational transformations. Indeed, in one direction, a sequent $A_{1}, \ldots, A_{n} \Rightarrow$ $B_{1}, \ldots, B_{m}$ in $\mathrm{GLC}_{R S}^{*}$ can be translated into a sequent of relations $A_{1} \leq$ $B_{1}|\ldots| A_{1} \leq B_{m}\left|A_{n} \leq B_{1}\right| \ldots \mid A_{n} \leq B_{m}$. One can easily check that the (translated versions of the) axioms and rules of $\mathrm{GLC}_{R S}^{*}$ are derivable in $\mathbf{R G}_{\infty}$. As an example we consider the following version of the rules $(\Rightarrow \supset(\supset))$ and $(\supset(\supset) \Rightarrow)$ of $\mathrm{GLC}_{R S}^{*}$, respectively:

$$
\frac{X \leq B \supset C|X \leq A \supset C| \mathcal{H}}{X \leq A \supset(B \supset C) \mid \mathcal{H}} \quad \frac{B \supset C \leq X|\mathcal{H} \quad A \supset C \leq X| \mathcal{H}}{A \supset(B \supset C) \leq X \mid \mathcal{H}}
$$


The above rules can be derived in $\mathbf{R} \mathrm{G}_{\infty}$ as follows (we omit side sequents that are not involved in the derivations):

$$
\begin{aligned}
& 1 \frac{C<A \mid A \leq C}{\leq C|C<A| A \leq C} \text { (weak.) } \frac{C \leq C}{C \leq C \mid A \leq C} \text { (weak.) }
\end{aligned}
$$

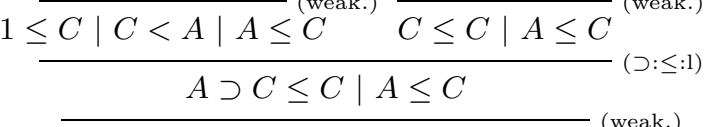

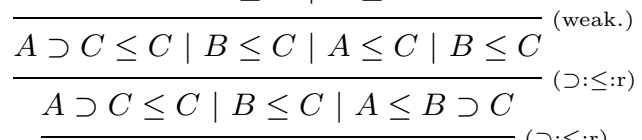

$$
\begin{aligned}
& \frac{X \leq B \supset C \mid X \leq A \supset C \quad \frac{A \supset C \leq B \supset C \mid A \leq B \supset C}{(\text { ( : } \leq: \mathrm{r})}}{\frac{X \leq B \supset C|A \leq B \supset C| X \leq B \supset C}{(\text { tr-cut })}}
\end{aligned}
$$

and

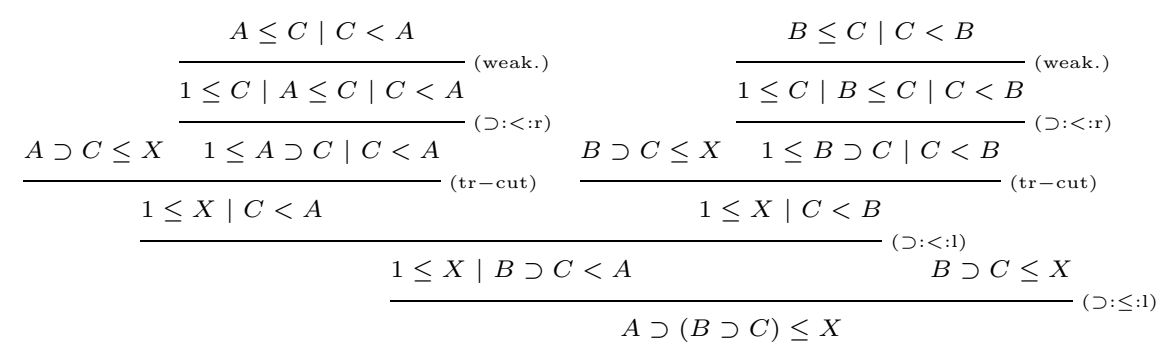

In the other direction, a sequent $G$ in $\mathbf{R G}_{\infty}$ can be translated into a sequent $\Gamma \Rightarrow \Delta$ in $\mathrm{GLC}_{R S}^{*}$ as follows (see Section 4 of [5]):

1. If $1 \leq B$ is a component of $G$, then $B \in \Delta$

2. If $B \leq 1$ is a component of $G$, then $B \supset B \in \Delta$

3. If $1<B$ is a component of $G$, then $B \supset B \in \Gamma$

4. If $B<1$ is a component of $G$, then $B \in \Gamma$

5. If $A \leq B$ is a component of $G(A, B \neq 1)$, then $A \supset B \in \Delta$

6. If $A<B$ is a component of $G(A, B \neq 1)$, then $B \supset A \in \Gamma$

One can easily check that this translation makes the axioms and rules of $\mathbf{R G}_{\infty}$ identical to those of $\mathrm{GLC}_{R S}^{*}$.

We emphasize that $\mathbf{R G}_{\infty}$ is just a special instance of a more general framework for theorem proving in many-valued logics. Our approach sheds light on the connections between $\mathrm{G}_{\infty}$ and other projective logics. In particular, it allows to understand the various formulations of "signed calculi" for finite-valued logics (see, e.g., [9]) as instances of the same methodological principle that is used to derive $\mathbf{R G}_{\infty}$. 


\section{References}

1. Avellone, A., Ferrari, M. and Miglioli, P.: Duplication-free tableau calculi together with cut-free and contraction free sequent calculi for the interpolable propositional intermediate logics. Logic J. of the IGPL, 7, (4) (1999) 447-480

2. Avron, A.: Hypersequents, logical consequence and intermediate logics for concurrency. Annals of Mathematics and Artificial Intelligence, 4 (1991) 225-248

3. Avron, A.: The method of hypersequents in the proof theory of propositional nonclassical logics. In Logic: from Foundations to Applications, European Logic Colloquium. Oxford Science Publications. Clarendon Press. Oxford (1996) 1-32

4. Avron, A.: A tableau system for Gödel-Dummett logic based on a hypersequential calculus. In Automated Reasoning with Tableaux and Related Methods (Tableaux'2000), volume 1847 of Lectures Notes in Artificial Intelligence (2000) 98-112

5. Avron, A. and Konikowska, B.: Decomposition proof systems for Gödel logics. Studia Logica, 69 (2001) 197-219

6. Baaz, M.: Infinite-valued Gödel logics with 0-1-projections and relativizations. In Gödel 96. Kurt Gödel's Legacy, volume 6 of LNL (1996) 23-33

7. Baaz, M., Ciabattoni, A. and Fermüller, C.: Cut-elimination in a sequentsof-relations calculus for Gödel logic. In International Symposium on Multiple Valued Logic (ISMVL'2001). IEEE (2001) 181-186

8. Baaz, M. and Fermüller, C.: Analytic calculi for projective logics. In Automated Reasoning with Tableaux and Related Methods (Tableaux'99), volume 1617 of Lectures Notes in Artificial Intelligence (1999) 36-51

9. Baaz, M., Fermüller, C. and Salzer, G.: Automated deduction for many-valued logic. In Handbook of Automated Reasoning. Elsevier (2001)

10. Baaz, M., Fermüller, C. and Zach, R.: Elimination of cuts in first-order finitevalued logics. J. Inform. Process. Cybernet. EIK, 29, (6) (1994) 333-355

11. Bachmair, L. and Ganzinger, H.: Ordered chaining for total orderings. In CADE'94, volume 814 of Lecture Notes in Computer Science (1994) 435-450

12. Bachmair, L. and Ganzinger, H.: Ordered chaining calculi for first-order theories of transitive relations. J. of the ACM, 45, (6) (1998) 1007-1049

13. Ciabattoni, A. and Ferrari, M.: Hypersequent calculi for some intermediate logics with bounded Kripke models. J. of Logic and Computation, 2, (11) (2001) 283-294

14. Dummett, M.: A propositional logic with denumerable matrix. J. of Symbolic Logic, 24 (1959) 96-107

15. Dunn, J.M. and Meyer, R.K.: Algebraic completeness results for Dummett's LC and its extensions. Z. Math. Logik Grundlagen Math, 17 (1971) 225-230

16. Dyckhoff, R.: A deterministic terminating sequent calculus for Gödel Dummett logic. Logic Journal of the IGPL, 7 (1999) 319-326

17. Gentzen, G.: Untersuchungen über das logische Schliessen I, II. Mathematische Zeitschrift, 39 (1934) and (1935) 176-210 and 405-431

18. Gödel, K.: Zum intuitionistischen Aussagenkalkül. Anz. Akad. Wiss. Wien, 69 (1932) 65-66

19. Hájek, P.: Metamathematics of Fuzzy Logic. Kluwer (1998)

20. Hájek, P., Godo, L. and Esteva, F.: A complete many-valued logic with productconjunction. Archive for Mathematical Logic, 35 (1996) 191-208 
21. Lukasiewicz, J.: Philosophische Bemerkungen zu mehrwertigen Systemen der Aussagenlogik. Comptes Rendus de la Societé des Science et de Lettres de Varsovie (1930) 51-77

22. Moisil, G.: Essais sur les logiques non chrysipiennes. Editions de l'Academie de la Republique Socialiste de Roumanie, Bucarest, 1972.

23. Rousseau, G.: Sequents in many valued logic I. Fund. Math., 60 (1967) 23-33

24. Schütte, K.: Proof Theory. Springer, Berlin and New York (1977)

25. Sonobe, O.: A Gentzen-type formulation of some intermediate propositional logics. J. of Tsuda College, 7 (1975) 7-14

26. Takahashi, M.: Many-valued logics of extended Gentzen style I. Science Reports of the Tokyo Kyoiku Daigaku, 9 (1967) 95-116

27. Takeuti, G. and Titani, T.: Intuitionistic fuzzy logic and intuitionistic fuzzy set theory. J. of Symbolic Logic, 49 (1984) 851-866

28. Troelstra, A.S. and Schwichtenberg, H.: Basic Proof Theory. Cambridge University Press (1996)

29. Visser, A.: On the completeness principle: a study of provability in Heyting's Arithmetic. Annals of Math. Logic, 22 (1982) 263-295 